\title{
PERFORMANCE IMPROVEMENT FOR SMART ANTENNA SYSTEM LEAST SQUARE BEAMFORMING ALGORITHMS
}

\author{
${ }^{*}$ Aseel A. Qasim ${ }^{1}$
}

Adheed H.Sallomi ${ }^{2}$

1) M.Sc., Student Electrical Engineering Department, Mustansiriyah University, Baghdad,

2) Prof. Dr., Electrical Engineering Department, Mustansiriyah University, Baghdad, Iraq.

\begin{abstract}
Smart antenna is great prominence in many applications like radar and wireless communication. The adaptive algorithms are periodically updated the weight vector to monitor the desired signal sources in a timevarying environment by modifying the array pattern with nulls to the direction of the sources of interference. A major goal of this paper is to simulate different improvement aspect of conventional adaptive algorithms that has step size factor $(\mu)$ for spatial beamforming and normalizing the least square algorithm by SIR factor, here, simulations are carried out using a MATLAB system to adjust the weight value for better performance and computational complexity and to compare the characteristics of the algorithms. Also in this paper presents the comparison for the effeteness of two array geometry by calculating the BER factor for both and also the relation between the number of antenna for them and SNR factor. Regularly to defeat the computational complexity problem this paper would introduce a new investigation of achievements and a study of two enhanced beamforming algorithms: Least Mean Square Step Size Improvements (LMSSI) and Normalized Least Mean Square by signal to interference ratio improved (SIR-LMS), both algorithm are comparable in convergence rate. The LMSSI has a slower rate of convergence than the SIR-LMS, but much faster than a standard LMS algorithm.
\end{abstract}

Keywords: Smart antenna, least mean square (LMS), least mean square step size improvement (LMSSI), step size factor (SSF), signal to interference ratio (SIR), mean square error (MSE).

\section{Introduction}

Smart antenna system (SA) or an alternative way called "digital beamformed" arrays (DBF) or adaptive antenna arrays (when using adaptive algorithms) [1] is the combination of array elements with digital signal processing (DSP) techniques. The SA has a spirited role in increasing the desire services of the various mobile communication system to achieve wireless channel spatial parameters in noisy environments [2].

SA framework is a modern and economical wireless and mobile communication systems technology that has limited there capacity and performance by two major feebleness which is co-channel and multipath interference. Multipath is a condition that occurs when a signal is transmitted is reflected by different environmental hurdles. The SA receiver determines the signature of the spatial signal and calculates vector beamforming, tracking and guiding the main beam on the mobile target.

Array systems used in various applications like sonar, radar, seismology, radio astronomy, biomedicine, and wireless communication.

SA has many advantages such as increased system capacity reduced Bit Error Rate (BER), 
compatibility with multiple access techniques, improved beam path, decreased fading effects, decreased signal-to-interference ratio (SIR), and increased signal-to-noise ratio (SNR) [3],[4]. Many algorithm have been introduced for this purpose, generally, Least Mean Square (LMS) algorithm is commonly used because of reasonable stability properties, fairly robustness to compliance errors and, its low computational complexity [2]. However, there is a poor convergence rate in the LMS algorithm, which mines system performance. To improve the convergence rate, the LMS algorithm is updated by standardization, known as normalized LMS (NLMS).

There are many adaptive algorithms derived from the conventional LMS algorithm [5], [6] the optimum weights provide minimum MSE. The Variable Step Size (VSSLMS) algorithms seek to boost the LMS algorithm's convergence while maintaining stable state performance. The proposed Error Controlling Variable Step Size factor algorithm produced a new style of choosing the step size for the VSSLMS algorithms. Controlling Variable Step Size factor uses to improve the performance of the antenna array by modifying the step size of the NLMS algorithm.

The SIR-LMS combines the SIR and the proposed LMS algorithm, so in this aspect, two forms of algorithm would be jointed the fixed beam and the adaptive beam to improve the performance of an adaptive algorithm, such as convergence speed, weight stability, and interference suppression.

This paper illustrates the modeling of a communication system that includes the smart antenna system to display the enhancement in Received Power (SNR and BER), so creates Array Factor and guides the pattern for both Linear and Circular array, so that system can be considered as fixed beam system (conventional) just for generates the signal, modulates it and transmits it with added Noise through the channel.

\section{Mathematical Model}

\subsection{Bit Error Rate (BER)}

Fig.1 presented the flowchart of simulating for a communication system module that included transmitter of the signal message, a modulator for the signal, beamformer, transmission channel, receiver, demodulator, and sampler. It transmits and create a pattern in the direction of DOA and receive message signal as user/target and measure received signal quality and BER.

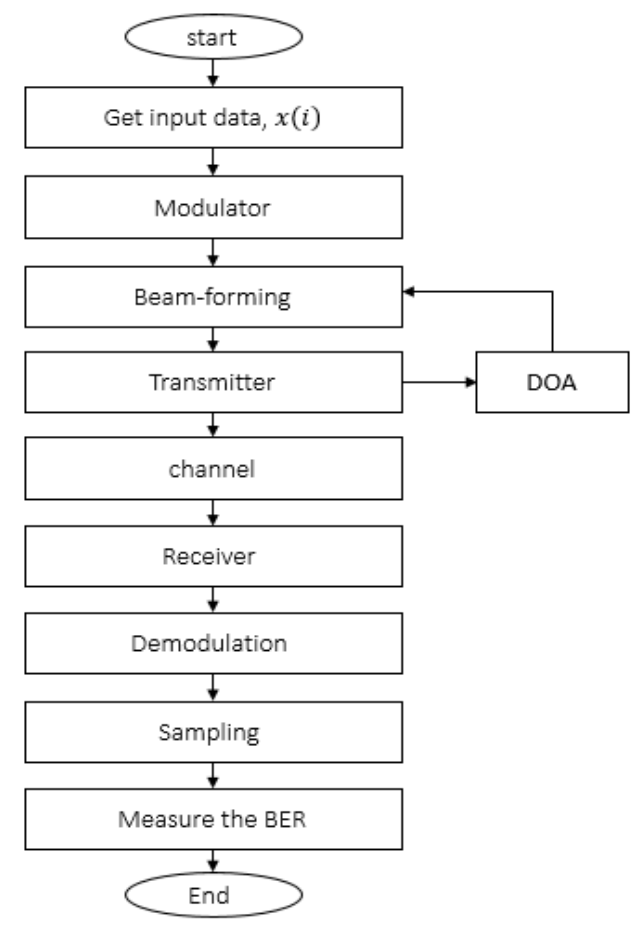

Figure 1. Processing flowchart

Different tests of common antenna arrays like linear and circular arrays are comparing and the overall system performance had been inspected along with changing some parameters. Testing BER and impact of an increasing number of elements in two types of an antenna array, linear and circular arrays besides the effect of increasing the noise value added to the system. The BER had been measured by sending and generating a random message of 1000 Bits using MATLAB program and comparing the received single with the transmitted one. Matrix of BER created to contains the total BER of the 1000 Bits 


\subsection{Least Mean Square (LMS) Algorithm}

Fig.2 illustrated the block diagram of the SA system.

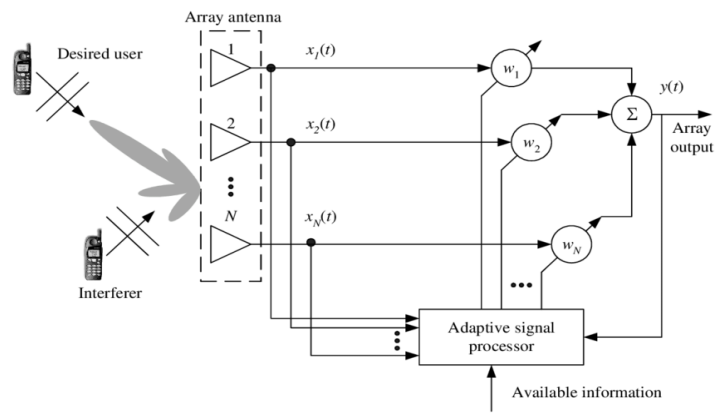

Figure 2. Block Diagram of Smart Antenna System.

The weight vector $\overline{\mathrm{w}}=\left[\begin{array}{llll}\mathrm{w}_{1} & \mathrm{w}_{2} & \ldots . \mathrm{w}_{\mathrm{M}}\end{array}\right]^{\mathrm{T}}$ as shown in Fig. 2 must be revised to minify the error while iterating the SA adaptive weights. The desired signal $\overline{\mathrm{S}}(j)$ and interferers $I_{1}(j), I_{2}(j), \ldots I_{N}(i)$ are received by each antenna array element $(\mathrm{M})$ with the same number of adaptive weights. Times are noted by the $j_{\text {th }}$ time samples.

$e(j)=d(j)-\bar{w}^{H}(j) \bar{x}(j)$

The squared error is

$|e(j)|^{2}=\left|d(j)-\bar{w}^{H}(j) \bar{x}(j)\right|^{2}$

The cost function is given as

$J(\bar{w})=D-2 \bar{w}^{H} \bar{r}+\bar{w}^{H} \bar{R} x x \quad \bar{w}$

Where $D=E\left[|\mathrm{~d}|^{2}\right]$

Taken the gradient of equation (5) and equate it to zero to reducing the cost function.

$\overline{\mathrm{w}}_{\mathrm{opt}}=\overline{\mathrm{R}} \mathrm{xx}^{-1} \overline{\mathrm{r}}$

The prompt estimates of these values are given as

$R_{x x} \approx \bar{x}(j) \bar{x}^{H}(j)$

$r(j) \approx d^{*}(j) \bar{x}^{H}(j)$

After the cost function gradient, the LMS weight vector would be $\bar{w}(j+1)=\bar{w}(j)+\mu e^{*}(j) \bar{x}(j)$

As appear in equation (9), the LMS algorithm convergence is proportionate in a direct way with step size factor $(\mu)$. Therefore, selecting a step size within arrange to ensure convergence is imperative [7], [8].

The convergence and changing angles of arrival signal should have the same instantaneous movement so if the convergence is slower, the SA can't achieve the track the changing signal in the signal of interest and that leads to on the inhibitory issue. In other words, if $\mu$ is prompt, the optimum weights of LMS would be overshoot. This is called the underdamped case because the weights will oscillate about the optimum weights, but the desired solution won't be tracked accurately. It is therefore imperative to choose a step-size within a range that compensates for convergence, such as

$0 \leq \mu \leq \frac{1}{2 \lambda_{\max }}$

Where $\lambda_{\max }$ represent the largest Eigenvalue of $\bar{R} x x$, if there is only one desired signal and the rest interfering signals are noise, equation (10) could be approximate as

$0 \leq \mu \leq \frac{1}{2 \operatorname{trace}\left[\hat{R}_{x x}\right]}$

Three main factors guided the response of the LMS algorithm which is, $\mu$, number of weights, and the Eigen-value of the correlation matrix of the input vector data.

Many iterations ought to before satisfying to order to achieve the convergence rate, that drawback of the conventional LMS because of using fixed $\mu$. Two types of antenna configuration would be used by traditional LMS algorithms but for simplicity, the improvements on this algorithm will be applied just on linear array antenna. 
2.3 Variable Step Size via Error Controlling Algorithm

Normalized LMS (NLMS) is modified to enhance the convergence rate, [9], [10]. There are several adaptive algorithms derived from the slandered LMS algorithm [11], [12]. The NLMS algorithm is a variable step size (VSSLMS) algorithm extension that selects a variable value of $\mu(\mathrm{n})$ for each algorithm iteration which is investigated concerning the inverse of the total expected energy of the SA incoming signal immediate values. The NLMS minifies the step size $\mu_{\text {NLMS }}(n)$ to make significant changes in updated weight vectors. That made the convergence algorithm more stable and faster than using a fixed $\mu[11]$. The variable $\mu(n)$ a factor of NLMS algorithm gave as [13]

$\mu_{\mathrm{NLMS}}(\mathrm{j})=\frac{\mu_{0}}{\|\overline{\mathrm{x}}(\mathrm{j})\|^{2}}$

Where $\mu_{0}$ between (0-1) is a small positive constant.

So, the weight vector would be updated as

$\overline{\mathrm{w}}(\mathrm{j}+1)=\overline{\mathrm{w}}(\mathrm{k})+\frac{\mu_{0}}{\|\overline{\mathrm{x}}(\mathrm{k})\|^{2}} \mathrm{e}^{*}(\mathrm{k}) \overline{\mathrm{x}}(\mathrm{k})$

Where

$e(j)=$ error signal

To avoid the denominator to be zero a small constant q should add [14].

$\bar{w}(j+1)=\bar{w}(j)+\frac{\mu_{0}}{q+\|\bar{x}(j)\|^{2}} e^{*}(j) \bar{x}(j)$

Another way for choosing the $\mu(n)$ is the error driving algorithm to improve the performance of the $\mathrm{SA}$ system by modifying $\mu_{\mathrm{NLMS}}(\mathrm{n})$.The small fixed $\mathrm{q}$ in the NLMS algorithm has a constant impact in the $\mu$ factor and can cause a decrease in its value and this decrease affects the weight stability and the NLMS algorithm's convergence rate. The error signal can be used to avoid null denominator in each iteration and to regulate the step size according to this method, you can set parameter $q$ as

$\mathrm{q}=\frac{\sigma_{\mathrm{n}}}{\|\mathrm{e}(\mathrm{j})\|^{2}}$

Where $\sigma_{\mathrm{n}}$ is the square root of the noise variance $\sigma_{\mathrm{n}}^{2}$. Thus, the step size could be written as

$\mu_{\mathrm{SS}}(\mathrm{j})=\frac{\mu_{0}}{\frac{\sigma_{\mathrm{n}}}{\|\mathrm{e}(\mathrm{j})\|^{2}}+\|\overline{\mathrm{x}}(\mathrm{j})\|^{2}}$

Therefore, the weight vector of the error control step size algorithm is

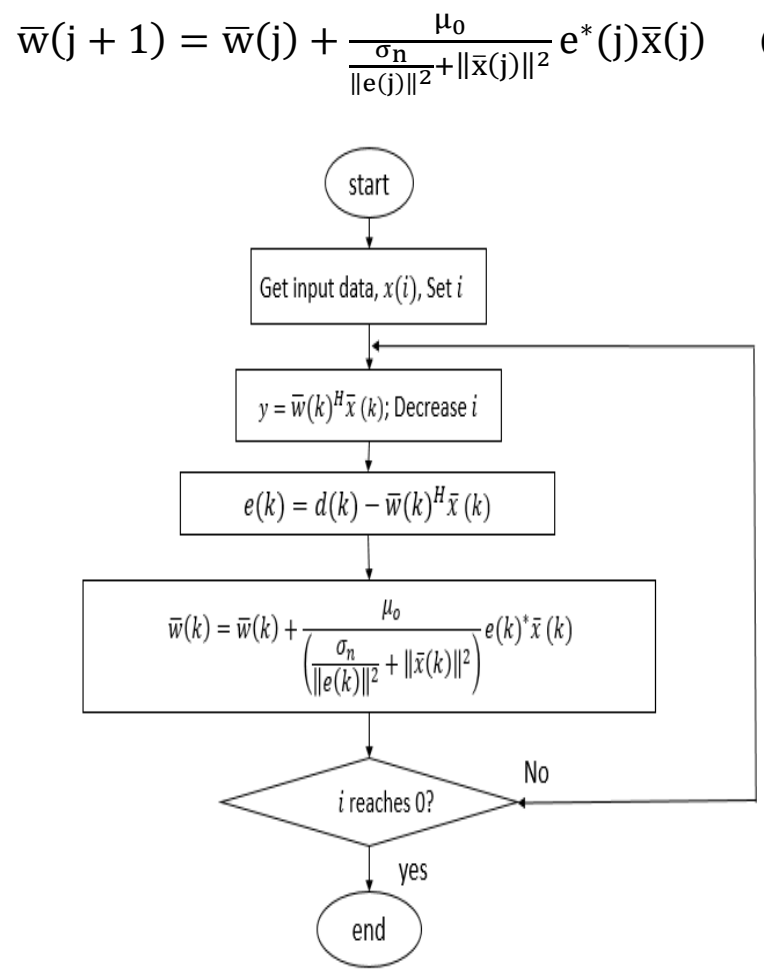

Figure 3. Flowchart for variable step size algorithm.

The $\mu(\mathrm{n})$, maximizing and minimizing depends on the error signal of the antenna array, and the weight vector is changed to detect any changes. That can explain as at the beginning the error signal is large of the adaptive process, $\mathrm{q}$ is small and $\mu(n)$ is large to reduce the error readily. However, in steady-state, when the error signal is small so step size is small and $\mathrm{q}$ is large to get a low level of maladjustment. 
2.4 The Implementation of Normalizing LMS Algorithm by Maximum SIR Factor

The most useful advantage of this algorithm converges quicker than any other adaptive algorithm.

The proposed adaptive beam-forming algorithm, LMS normalize by SIR is introduced here, which combines the maximum SIR and LMS algorithm, the maximization of the SIR is one criterion that could be applied to maximize the signal obtained and eliminate the interference signals. The SIR would automatically maximize if all interference cancels by putting nulls at their destination address that leads to improving the performance of an adaptive algorithm.

The SIR approaches are used to determine the best vectors of weights to each antenna element of the array by considering a fixed beam pattern instead of an arbitrary value before the actual weight coefficients are determined vectors by LMS. The weight coefficients calculated by normalization is set as the initial coefficients and then modified using standard LMS algorithms.

Both the desired signal and the undesired signals $\left(\bar{R}_{s S} \& \& \bar{R}_{u u}\right)$ array correlation matrices could be calculated .which called the array covariance matrices. However, the covariance matrix aims to remove the correlation matrix. Since the type of the system noise or the front end detector output does not mostly know, it is preferable to label all $\mathrm{R}$ matrices as correlation matrices.

The novel optimum updated weight vectors of combining the two aspects of $S I R_{\max }$ and LMS algorithm introduces according to the following equations:

The desired signal weighted output power is specified as

$\sigma_{s}^{2}=E\left[\left|\bar{w}^{H} \cdot \bar{x}_{s}\right|^{2}\right]=\bar{w}^{H} \cdot \bar{R}_{s S} \cdot \bar{w}$

$\bar{R}_{s S}=E\left[\bar{x}_{s} \bar{x}_{s}{ }^{H}\right]$

$=$ signal correlation matrix
The undesired signals weighted output power for is specified as

$\sigma_{u}{ }^{2}=E\left[\left|\bar{w}^{H} \cdot \bar{x}_{u}\right|^{2}\right]=\bar{w}^{H} \cdot \bar{R}_{u u} \cdot \bar{w}$

Where, $\bar{R}_{u u}=\bar{R}_{i i}+\bar{R}_{n n} \bar{R}_{i i}$,

is the interferers correlation matrix for

$\bar{R}_{n n}$, is the noise correlation matrix

The SIR described the ratio of the desired signal to the unfavorable signal.

$S I R=\frac{\sigma_{s}{ }^{2}}{\sigma_{u}{ }^{2}}=\frac{\bar{w}^{H} \cdot \bar{R}_{S S} \cdot \bar{w}}{\bar{w}^{H} \cdot \bar{R}_{u u} \cdot \bar{w}}$

By occupation the derivative regard to $\bar{w}$ and tuning the result equal to zero. The equation would be derived as

$\bar{R}_{u u}{ }^{-1} \bar{R}_{s s} \cdot \bar{w}=S I R \cdot \bar{w}$

Where the SIR being the eigenvalues, and $S I R_{\max }$ is equal to the largest eigenvalue $\lambda_{\text {max }}$ for the Hermitian matrix $\bar{R}_{u u}, \bar{R}_{s s}$

The optimum weight vector $\bar{w}_{\text {opt }}$ are the eigenvector associated with the largest eigenvalue, so

$\bar{R}_{u u}{ }^{-1} \bar{R}_{S S} \cdot \bar{w}_{S I R}=\lambda_{\text {max }} \cdot \bar{w}_{o p t}$

$=S I R_{\max } \cdot \bar{w}_{S I R}$.

The weight vector can pose in terms of the optimum Wiener solution.

$\bar{w}_{S I R}=\beta \cdot \bar{R}_{u u}{ }^{-1} \cdot \bar{a}_{0}$

Where, $\beta=\frac{E\left[|s|^{2}\right]}{S I R_{\max }} \cdot \bar{a}_{0}{ }^{H} \cdot \bar{w}_{S I R}$

And, the correlation matrix is defined as

$\bar{R}_{s S}=E\left[|s|^{2}\right] \bar{a}_{0} \cdot \bar{a}_{0}{ }^{H}$

The part that relates to the LMS algorithm was given in part (2). The proposed algorithm can be drawn in the flowchart as indicated in Fig.4. 


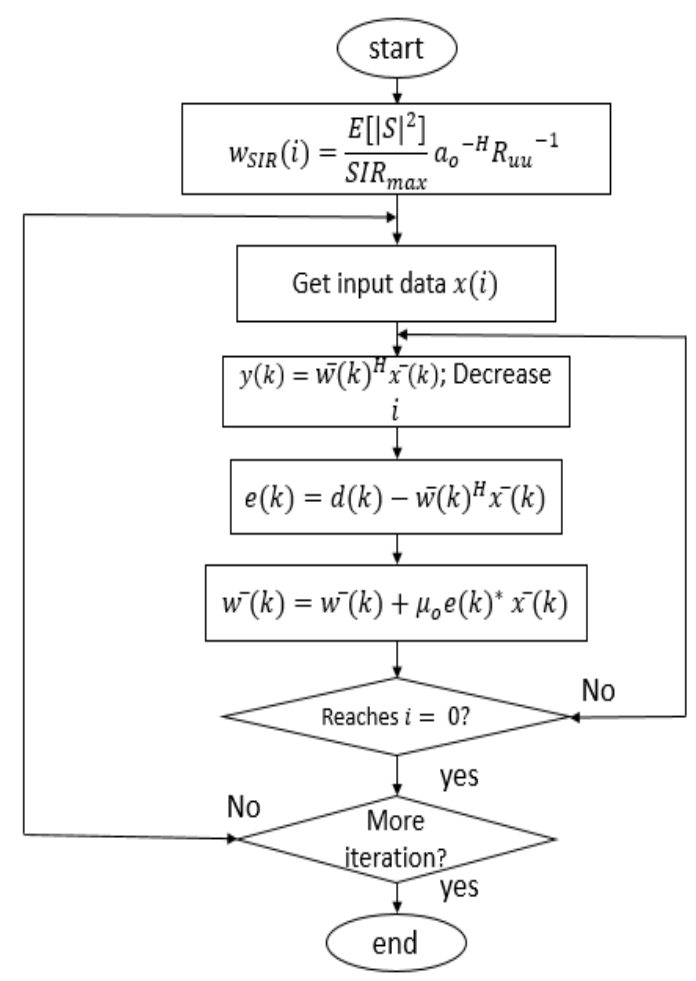

Figure 4. Normalized LMS algorithm by maximum SIR factor.

\section{Simulation Result:}

\subsection{Bit Error Rate (BER)}

In this section, the estimated BER had been measured, by sending a message of 1000 Bit in a random way using MATLAB program and comparing the received single with the transmitted on with AWGN as a source of noise in the channel.

This section studied the effect of the number of elements on the overall system performance. Fig.5 shows at lower noise values there are no errors when receiving a signal message however, errors occur when the noise starts to reach high levels and start to increase by increasing values of noise giving much bigger error values.

Fig.5 shows the comparison of the different number of elements $(\mathrm{m}=2,4,8,16)$ and shows that with two elements gives a lot of errors than using 8 elements in the array as so on when the number of elements increased, better results are given.

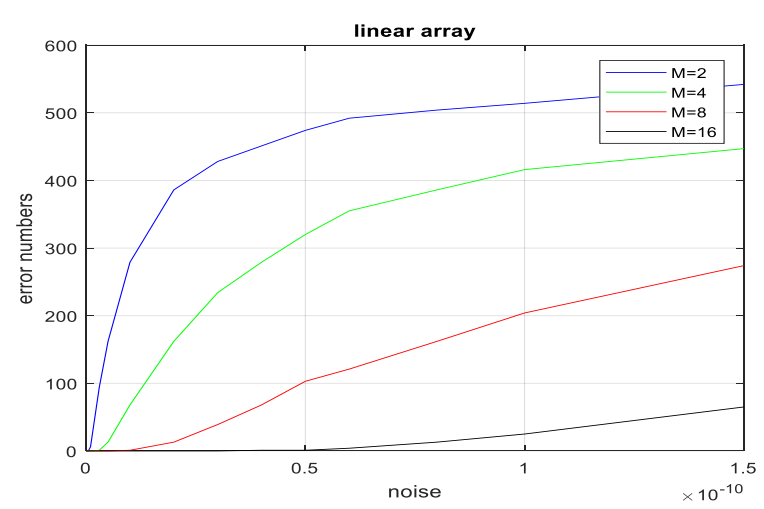

Figure 5. BER curve for different number of the linear array

The circular array represents 2D antenna arrays and it's also very resistive to noise and the BER is close to that of the linear array approximately with the same number of elements $(M=4,8,16)$ as shown in Fig.6

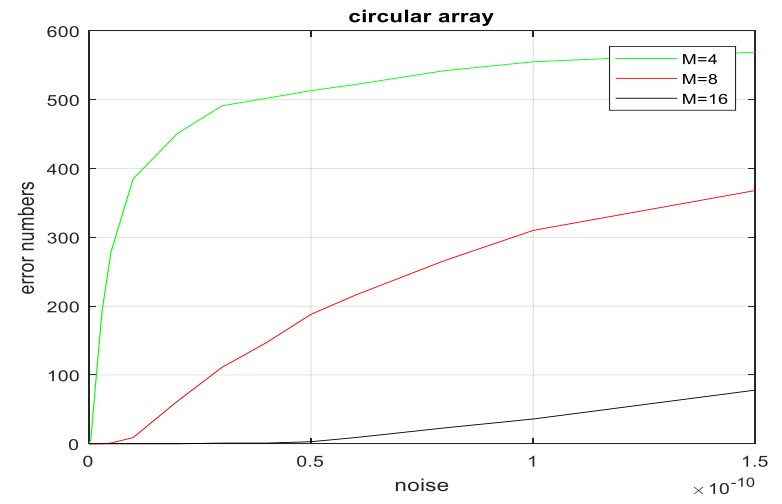

Figure 6. BER curve for different number of the circular array

Table 1 and fig. 7 shows the result value with 8 elements for linear and circular arrays.

Table 1. Number of errors concerning noise

$\begin{array}{ccc}\begin{array}{c}\text { Noise }(1 * \\ \left.10^{-16}\right)\end{array} & \begin{array}{c}\text { No. error of } \\ \text { linear array }\end{array} & \begin{array}{c}\text { No. error of } \\ \text { circular array }\end{array} \\ 1 & 0 & 0 \\ 5 & 0 & 0 \\ 10 & 0 & 0 \\ 50 & 0 & 0 \\ 100 & 0 & 0 \\ 500 & 0 & 0\end{array}$




\begin{tabular}{ccc}
1000 & 0 & 0 \\
5000 & 0 & 0 \\
10000 & 0 & 0 \\
30000 & 0 & 0 \\
50000 & 0 & 1 \\
100000 & 1 & 9 \\
200000 & 13 & 62 \\
300000 & 39 & 111 \\
400000 & 68 & 142 \\
500000 & 103 & 188 \\
600000 & 121 & 216 \\
800000 & 162 & 266 \\
1000000 & 204 & 310 \\
1500000 & 274 & 386 \\
\hline
\end{tabular}

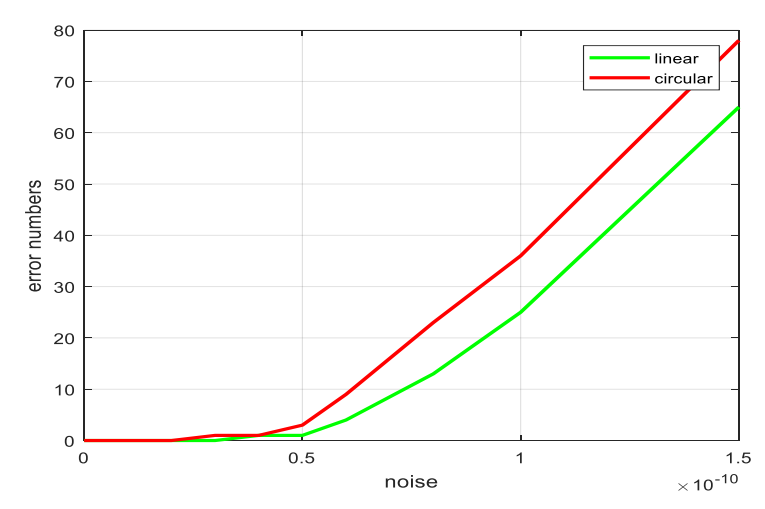

Figure 7. BER curve for linear and circular antenna array geometry with 8 elements

\subsection{Signal to Noise Ratio (SNR)}

The output results of the SNR with applying noise of $1 * 10^{-16}$ for 1000 Bits message signal to test the benefit of changing the element numbers on SNR values, are given in Table 2

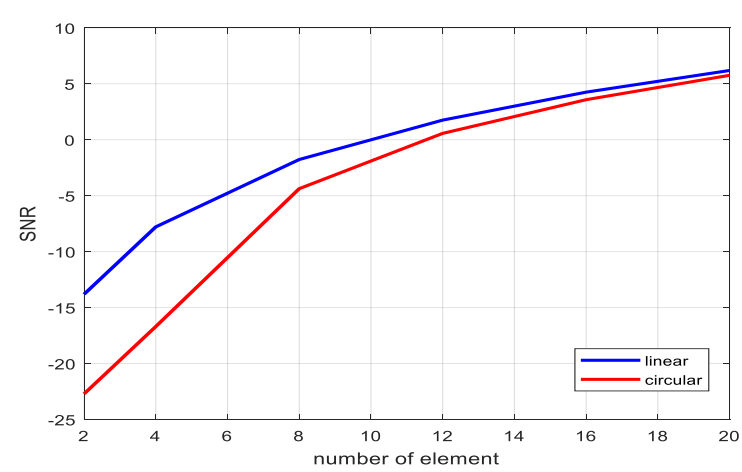

Figure 8. The relation between Number of elements and SNR (dB) for a linear and circular array

Table 2. SNR to the different number of elements

\begin{tabular}{ccc}
\hline $\begin{array}{c}\text { Number of } \\
\text { elements }\end{array}$ & SNR $(\mathrm{dB})$ & SNR $(\mathrm{dB})$ \\
& Linear array & Circular array \\
2 & -13.8067 & -22.7126 \\
4 & -7.7861 & -16.6925 \\
8 & -1.7655 & -4.3701 \\
12 & 1.7563 & 0.5706 \\
16 & 4.2551 & 3.5825 \\
20 & 6.1933 & 5.7610 \\
\hline
\end{tabular}

Fig.7 and Table 2 show that there is a small difference in the SNR curve for linear and circular array and it is clear to observe that the SNR curve of overall system increase with the number of elements used in the array regardless of the geometry of antenna array.

\subsection{Performance of Smart Antenna System via Variable Step Size Aspect}

This slit gives the achievements of proposed $\mu(n)$ improvement algorithm. In this simulation, the type of algorithm that chose to improve is the LMS algorithm, and it is worth mentioning that this proposed improvement could apply in any algorithm had step size factor $(\mu)$, the number of sample intervals applied equally to $(\mathrm{K}=100)$. Fig.9 produced the linear and polar plots of the array pattern in $\mathrm{dB}$ for the LMSSI algorithm. The 
radiation pattern of LMSSI algorithm is shown the main beam pattern of the system here steers the direction at $0^{0}$.

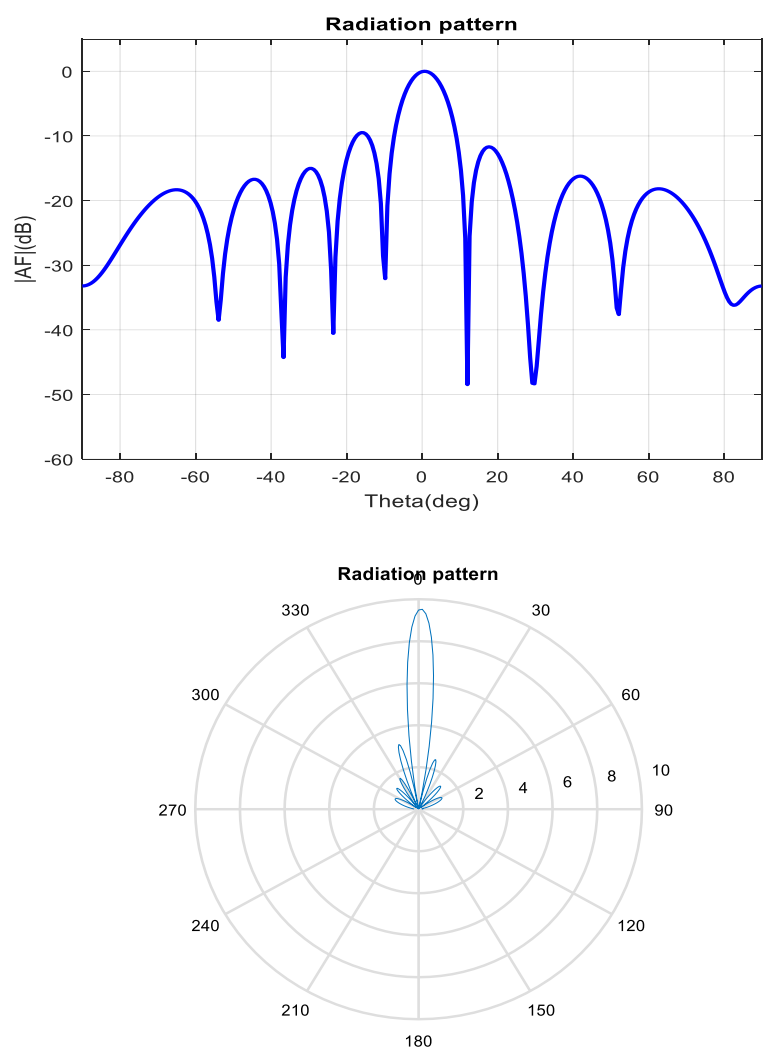

Figure 9. Linear \& polar plots, for radiation pattern of LMSSI algorithm at the number of element $\mathrm{M}=10$, DOA at 0 , and DOA interference at 30,-10.

The proposed step size algorithm in Fig.10 converges fast and without fluctuation compared with the LMS algorithms. On the other hand, the variation of weight values using the SSF algorithm in the steady-state is more stable than LMS in converging weights. From Fig.10, it can be observed that, the number of samples needed for variable $\mu(n)$ algorithms to converge are much lower than those for the LMS algorithm.

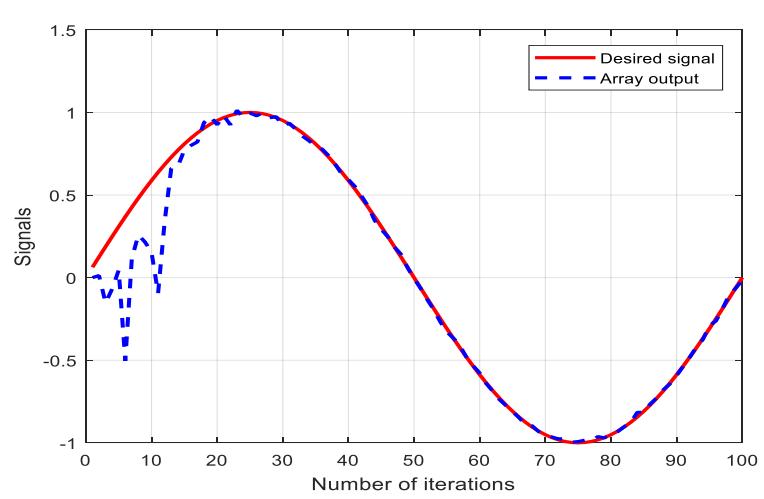

(a)

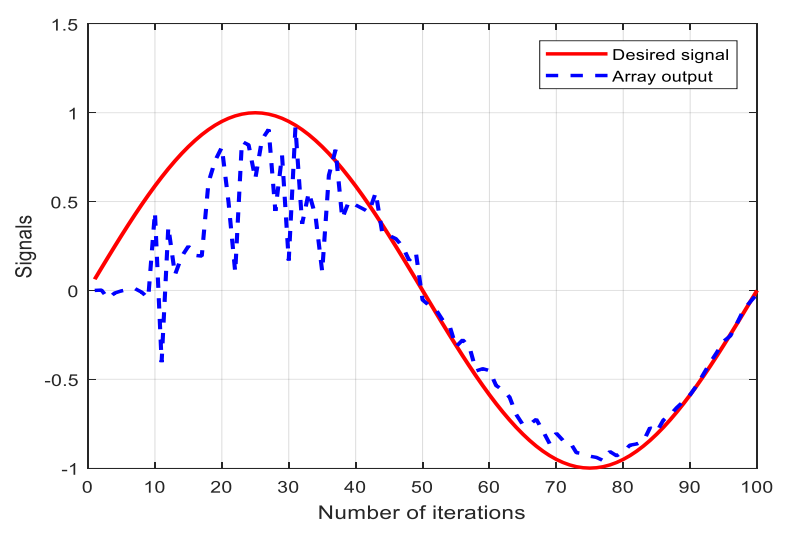

(b)

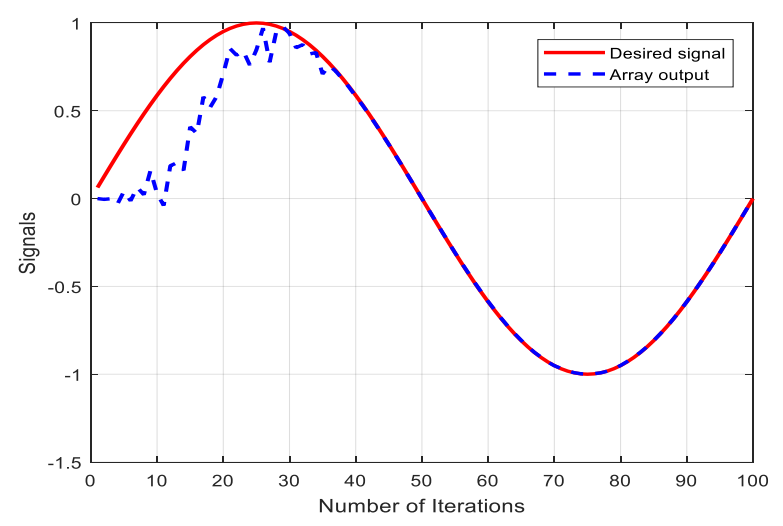

(c)

Figure 10. Tracking of the desired signal for (a) LMSSI (b) LMS algorithms for linear array (c) LMS algorithms for a circular array

Fig.10 displays the array output obtains and tracks the desired signal after 80 iterations for the linear array with conventional LMS and about 37 iterations just by changing the configuration to 
the circular array and about 22 iterations for variable step size and NLMS algorithms.

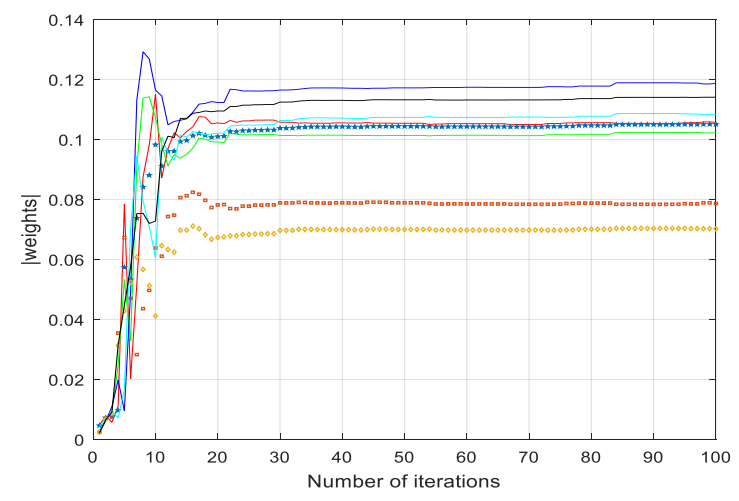

Figure 11. The magnitude of linear array weights for LMSSI algorithm

The proposed algorithm LMSSI reduces and increases the step size $\mu_{\mathrm{SS}}(\mathrm{k})$ to track any changes in the smart antenna environment.

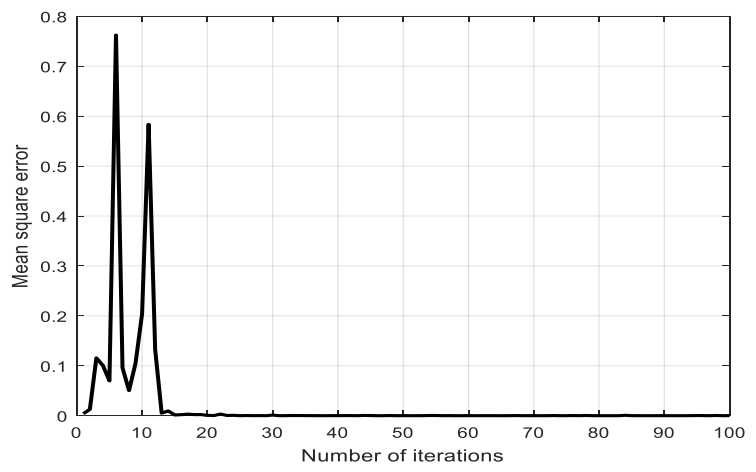

Figure 12. Mean square error curves for LMSSI

Moreover, from the MATLAB software, at the steady-state, the LMS error is almost $4.5028 \mathrm{e}-04$ whereas the LMSSI error is almost $2.7274 \mathrm{e}-04$ at around 100 iterations.

\subsection{Performance of Smart Antenna System via SIR-LMS Algorithm}

The SIR-LMS algorithm is developed for SA application which combined the individual pretty side of maximum SIR and LMS algorithms. This algorithm presented when the number of samples applied is $(\mathrm{K}=100)$. The linear and polar radiation pattern plots for the SIR-LMS algorithms are shown in Fig.13. This figure shows the null depth provided by the SIR-LMS at $-61.5 \mathrm{~dB}$. As shown in this Fig. 13 the main beam pattern of the system steers the direction at $0^{0}$
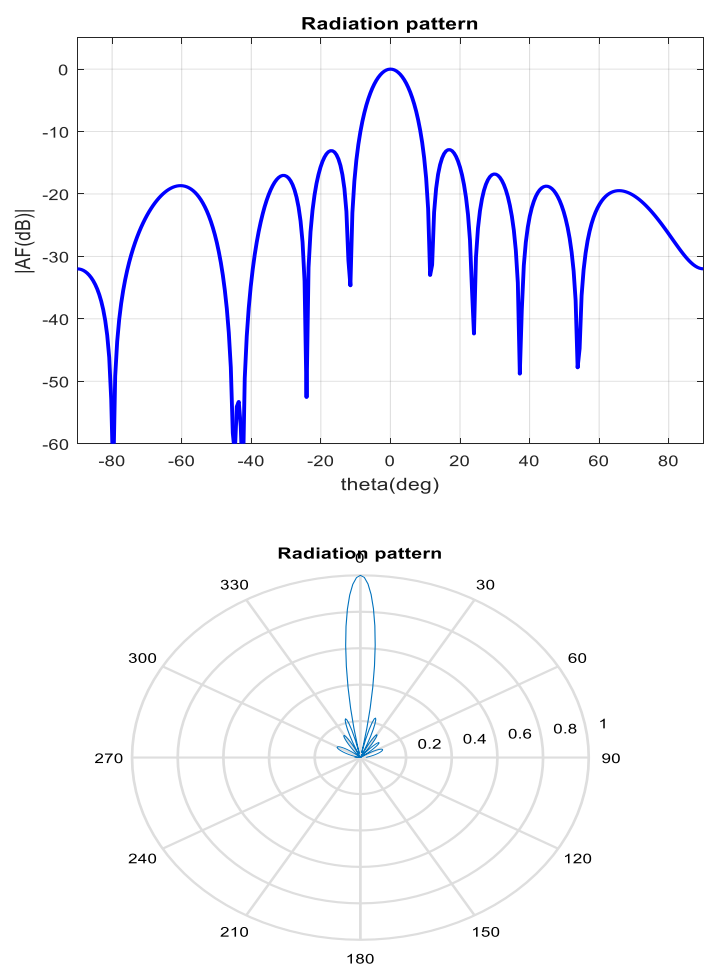

Figure 13. Linear \& polar plots, for radiation pattern of LMSSI algorithm at the number of element $\mathrm{M}=10$, $\mathrm{DOA}=0^{\circ}$, and DOA interference at $-45^{\circ}$.

Fig.14 illustrates the MSE curve for the SIRLMS algorithm.

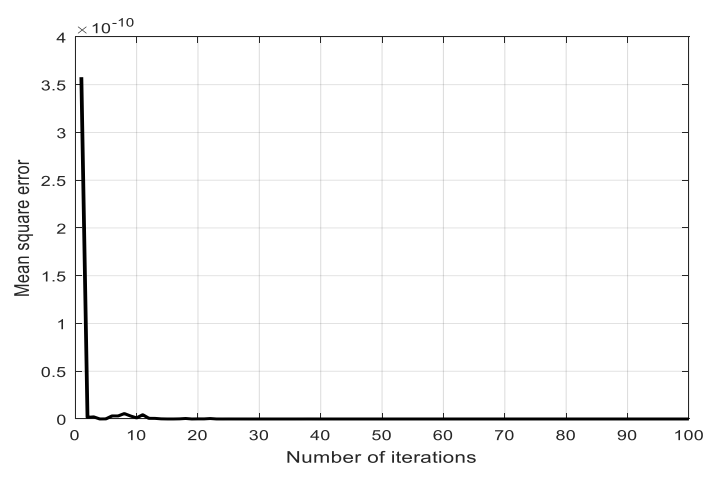

Figure 14. Mean square error curves for LMSIR algorithm 
Comparison with some algorithms like conventional LMS and NLMS algorithms start to converge from iteration numbers 65 and 25 respectively, whereas the converge in the SIRLMS algorithm starts to from the primer or initial stat of iterations. In this case, the SIR-LMS error is almost 7.2038e-28 with zero iteration number.

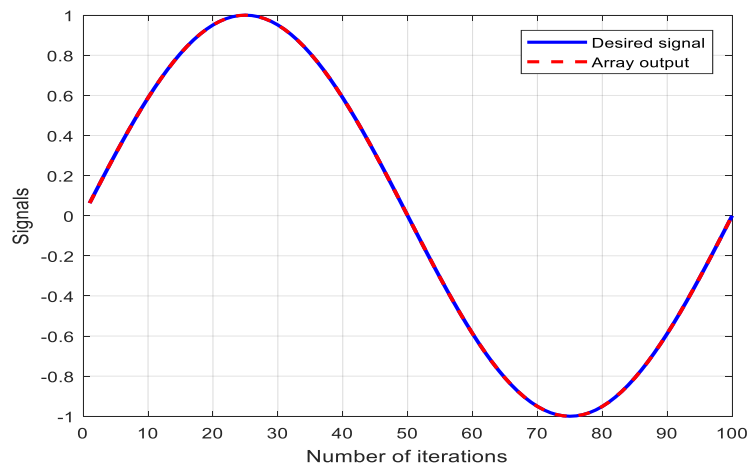

Figure 15. Tracking of the desired signal

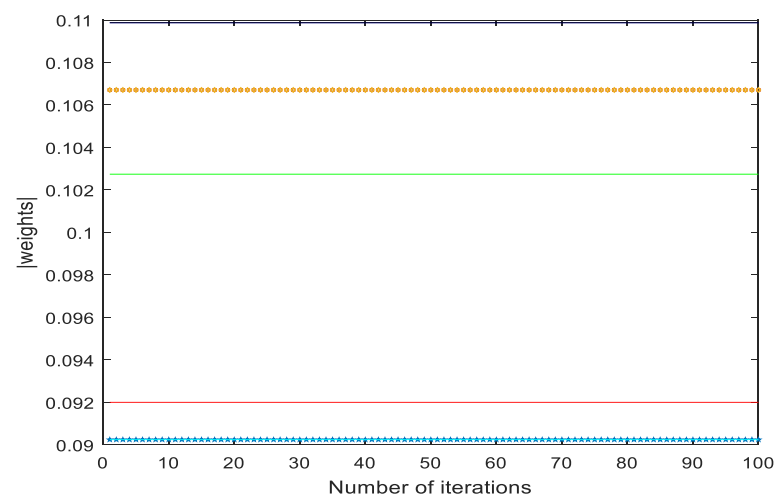

Figure 16. Magnitude weights for LMSIR algorithm

Figs. 15 \& 16 display that, The SIR-LMS start adjusting from the initial weight vector values to optimal weights. On the opposite, an algorithm such as LMS, NLMS ... etc. is beginning to converge from arbitrary weight towards optimum weight values. The simulation results illustrated that the proposed SIR-LMS algorithm delivers noticeable improvements in the elimination of interference, the convergence rate, and weight stabilizing which quickly reach their optimum values and without fluctuation compared with the other algorithms like LMS and NLMS algorithms.

\section{Conclusion}

This paper specialized by study two types of refinement which could by apply to any conventional least square algorithm for the linear and circular array to enhance the performance from the side of convergence rate and speed, MSE value, interference null, and numbers of iteration. Whence the first aspect study the effect of applying variable step-size factor by normalizing the stationary step size by the magnitude of signal error vector to ensure more stable and faster algorithm because of principle work by this algorithm to choose the appropriate value of $(\mu)$ to speed up and slow down the adaptive weight vector value to shorten the time when the adaptive process gets started. The second improvement has been done by joining the performance of fixed and adaptive beamforming algorithms, so firstly imposed the user was fixed to reach maximum SIR then insert to adaptation process and that leads to detect most fast and staple antenna array algorithm with minimum MSE vale and initially adaption process.

\section{Acknowledgments}

This work is supported by the Electrical Department/ Faculty of Engineering/ Mustansiriyah University.

\section{Conflict of interest}

The authors whose listed their names in this paper certify that they have NO affiliations with or involvement in any organization or entity with any financial interest in the subject matter or materials discussed in this manuscript. 


\section{References}

1. B. Xie, "Partial Update Adaptive Filtering." Virginia Tech, 2011.

2. F. Gross, "Smart antennas for wireless communications with MATLAB," McGraw Hills, 2005.

3. C. A. Balanis and P. I. Ioannides, "Introduction to smart antennas," Synth. Lect. Antennas, vol. 2, no. 1, pp. 1-175, 2007.

4. L. C. Godara, "Application of antenna arrays to mobile communications. II. Beam-forming and direction-of-arrival considerations," Proc. IEEE, vol. 85, no. 8, pp. 1195-1245, 1997.

5. R. S. Kawitkar and D. G. Wakde, "Smart antenna array analysis using LMS algorithm," in 2005 IEEE International Symposium on Microwave, Antenna, Propagation and EMC Technologies for Wireless Communications, 2005, vol. 1, pp. 370-374.

6. A. D. Poularikas and Z. M. Ramadan, Adaptive filtering primer with MATLAB. CRC Press, 2017.

7. Z. Rong, "Simulation of adaptive array algorithms for CDMA systems." Virginia Tech, 1996.

8. I. Stevanovic, A. Skrivervik, and J. R. Mosig, "Smart antenna systems for mobile communications," 2003.

9. M. A. Haque and M. K. Hasan, "Variable step-size multichannel frequency-domain LMS algorithm for blind identification of finite impulse response systems," IET Signal Process., vol. 1, no. 4, pp. 182-189, 2007.

10. S. Hadei, "A family of adaptive filter algorithms in noise cancellation for speech enhancement," arXiv Prepr. arXiv1106.0846, 2011
11. S. Hossain, M. T. Islam, and S. Serikawa, "Adaptive beamforming algorithms for smart antenna systems," in 2008 International Conference on Control, Automation and Systems, 2008, pp. 412-416.

12. S. Jimaa, "Convergence evaluation of a random step-size NLMS adaptive algorithm in system identification and channel equalization," Adapt. Filter., p. 1, 2011.

13. B. KRSTAJIĆ, L. STANKOVIĆ, and Z. USKOKOVIĆ, "A Variable Step-Size LMS Algorithm."

14. M. Z. U. Rahman, V. A. Kumar, and G. V. S. Karthik, "A low complex adaptive algorithm for antenna beam steering," in 2011 International Conference on Signal Processing, Communication, Computing and Networking Technologies, 2011, pp. 317321. 